\title{
Répartition spatio-temporelle des températures dans l'agglomération de Kairouan (Tunisie) pendant la saison chaude 2019
}

\author{
M. Chebli ${ }^{12 *}$, S. Charfi ${ }^{1}$ et S. Dahech ${ }^{12}$ \\ ${ }^{1}$ Université de Sfax, Faculté des Lettres et Sciences Humaines, Laboratoire SYFACTE, Sfax, Tunisie \\ ${ }^{2}$ Université de Paris, UMR PRODIG, Paris, France
}

\begin{abstract}
Résumé - L'effet de site et la nature du substrat modifient la température de l'air dans l'agglomération de Kairouan par rapport à son environnement rural, donnant naissance à un îlot de chaleur urbain (ICU) d'une intensité moyenne d'environ $4^{\circ} \mathrm{C}$. Cette étude visant à cerner la variation spatio-temporelle des températures pendant la période estivale est menée à l'aide de mesures fixes et itinérantes. L'étude montre que l'intensité de $l^{\prime} \mathrm{ICU}$ varie de 2 à $9^{\circ} \mathrm{C}$ en fonction des types de temps. Les zones agricoles et vallonnées au nord-ouest de l'agglomération sont les moins chaudes. Ce travail montre des différences entre la variation spatiotemporelle de l'ICU et celle du confort thermique : dans le centre-ville, l'air sec fait baisser les températures ressenties.
\end{abstract}

Mots-clés : Kairouan (Tunisie) / îlot de chaleur urbain / mesures fixes / mesures itinérantes / confort thermique.

\begin{abstract}
Spatio-temporal temperature spread in the Kairouan agglomeration (Tunisia) during the summer of 2019. The site effect and the substratum nature affect the air temperature in Kairouan agglomeration compared to its rural environment, resulting in an urban heat island increase (UHI) of an average intensity of about $4^{\circ} \mathrm{C}$. This study, which aimed to identify the spatio-temporal variation of temperatures during the summer period, is carried out using fixed and car survey measurements. The study shows that the intensity of the (UHI) ranges between 2 and $9^{\circ} \mathrm{C}$ depending on the time. In fact, there is a sharp drop in agricultural and hilly areas. This work shows that there are differences between the spatial-temporal variation of the UHI and that of thermal comfort: in the town center, dry air lowers the real feel.
\end{abstract}

Keywords: Kairouan (Tunisia) / urban heat island / fixed measurements / car survey / thermal comfort.

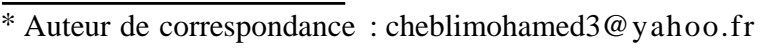

\section{Introduction}

L'augmentation continue de la température à l'échelle globale s'est accompagnée, durant les trois dernières décennies, d'une extension urbaine démesurée et d'une prolifération des activités anthropiques et de problèmes environnementaux en ville (Yang et al., 2016; Mathew et al., 2018). L'extension et la densification rapide des villes se manifestent et se répercutent par la croissance des surfaces imperméables, l'intensification du trafic routier, une pollution atmosphérique accrue et des émissions de chaleur anthropogénique entraînant une accumulation de chaleur (Qiuet et al., 2017). A titre d'exemple, selon Djen (1992), parmi les conséquences de l'expansion urbaine, Shanghai mégalopole qui a vu sa surface urbanisée doubler entre 1950 et 1980 - a enregistré une hausse de $0,7^{\circ} \mathrm{C}$ de l'écart entre la température minimale du centre-ville et celle de sa campagne, une diminution de la vitesse du vent de 3,2 à 2,9 m/s, une hausse du pourcentage du rayonnement diffus de 47 à $59 \%$ et 
une faible diminution de l'humidité relative. Les modifications du climat de la ville se traduisent par un îlot de chaleur urbain (ICU). Ce phénomène désigne une anomalie positive de la température de l'air entre les zones urbaines et leurs alentours ruraux (Oke, 1988 ; Dahech, 2007 ; Carrega, 2013 ; Leconte, 2014 ; Charfi, 2018 ; Mathew et al., 2018). Ce phénomène est très fréquent et intense par temps radiatif, avec ciel clair ou faible nébulosité et un vent faible ou nul (Cantat, 2004 ; Dahech, 2014). La configuration spatiale de l'ICU est influencée par les caractéristiques de la ville tels que la taille et la géométrie, le site, la latitude, l'éloignement de la mer et la situation météorologique (Wonga et al., 2011 ; Serrano et al., 2015).

Les effets néfastes de l'ICU englobent l'augmentation de la consommation d'énergie électrique et d'eau potable (Dahech et Ghribi, 2017) et les émissions des gaz à effet de serre. Les conséquences sanitaires de l'ICU deviennent plus graves par temps caniculaire (Hendel et al., 2017).

Sur la rive sud de la Méditerranée, la fréquence du beau temps en été, favorable à la mise en place d'un ICU intense, est très élevée à cause de la forte subsidence dynamique (El Melki, 2010). Dans les villes tunisiennes, la température de l'air nocturne augmente pendant la saison chaude de façon remarquable. La température minimale quotidienne dépasse souvent $25^{\circ} \mathrm{C}$ notamment au sud et à l'intérieur du pays. Les études antérieures concernant l'ICU s'intéressent uniquement à des villes littorales telles que Bizerte (Dahech et Charfi, 2017), Tunis (Charfi, 2012) et Sfax (Dahech, 2007).

Dans cette étude, nous nous intéressons au champ thermique de la ville de Kairouan qui n'a jamais été abordée malgré ses particularités thermiques (température élevée en été), géographiques (ville de l'intérieur chaude et sèche, avec une topographie vallonnée), et urbaines (ville médiévale de taille moyenne qui connait un étalement urbain remarquable souvent par le biais de constructions informelles) et sociales (taux de pauvreté le plus élevé en Tunisie), d'où une population plus vulnérable face à la forte chaleur.

Ce travail a pour objectif de caractériser la répartition spatio-temporelle de la température à Kairouan en mettant l'accent sur l'ICU, et d'étudier son impact sur le confort thermique pendant l'été 2019.

\section{L’agglomération de Kairouan : cadre humain et topoclimatique}

\subsection{Etalement urbain, population et occupation du sol}

L'étalement de l'emprise urbain de Kairouan, favorisé par une topographie peu accidentée, s'est fait dans toutes les directions et à un rythme très soutenu. La superficie bâtie est passée de 150 hectares en 1956 à presque 2300 hectares actuellement (Nasrallah, 2019).

Cette ville compte plus de 200000 habitants (INS, 2014). L'agglomération prend la forme d'un cercle de $5 \mathrm{~km}$ de rayon, caractérisée par un tissu urbain relativement dense dans sa partie centrale (figure 1). La densité urbaine dépasse en moyenne $7990 \mathrm{hab} / \mathrm{km}^{2}$ et frôle le double dans les secteurs populaires denses comme «EL Menchia » à l'ouest de l'agglomération. Les bâtiments dans la partie centrale de la ville sont à la fois à vocation résidentielle, commerciale et administrative. Les quartiers populaires «sont regroupés essentiellement tout au long des principales voies de communications tels que le quartier de Ali Bey ( sur la RN 12 reliant Kairouan à Haffouz), Menchia qui s'allonge sur la route régionale $n^{\circ} 99$ reliant Kairouan à El Baten et quartier Bourji développé de part et d'autre de la route régionale $n^{\circ} 87$ reliant Kairouan à Ouled Chamekh»(Nassrallah, 2019). Les parcs et les jardins sont peu présents dans la ville. La végétation devient abondante dans la zone périphérique : il s'agit de terres agricoles, en bonne partie irriguées, dont l'exploitation change selon les saisons.

\subsection{Cadre topoclimatique}

La ville de Kairouan est située en Tunisie Centrale en bordure sud-est de la dorsale tunisienne, sur une plaine d'épandage à $50 \mathrm{~km}$ du littoral (Nassrallah, 2019). Cette plaine, localisée au nord de la région naturelle des basses steppes, forme une vaste cuvette de $100 \mathrm{~km}$ de long (axe nord-sud) et de $40 \mathrm{~km}$ de large (axe est-ouest) et est parcourue par un réseau hydrographique assez dense. La 
région, au relief modeste, possède une pente générale orientée ouest-est de 150 à 40 m d'altitude (figure 2A). Les profils topographiques réalisés (figure 2B) à partir des données SRTM renseignent sur les caractéristiques du site de l'agglomération de Kairouan. Le profil A-B montre une concavité au niveau de la zone agglomérée, par contre le profil C-D possède une pente orientée vers le nord.
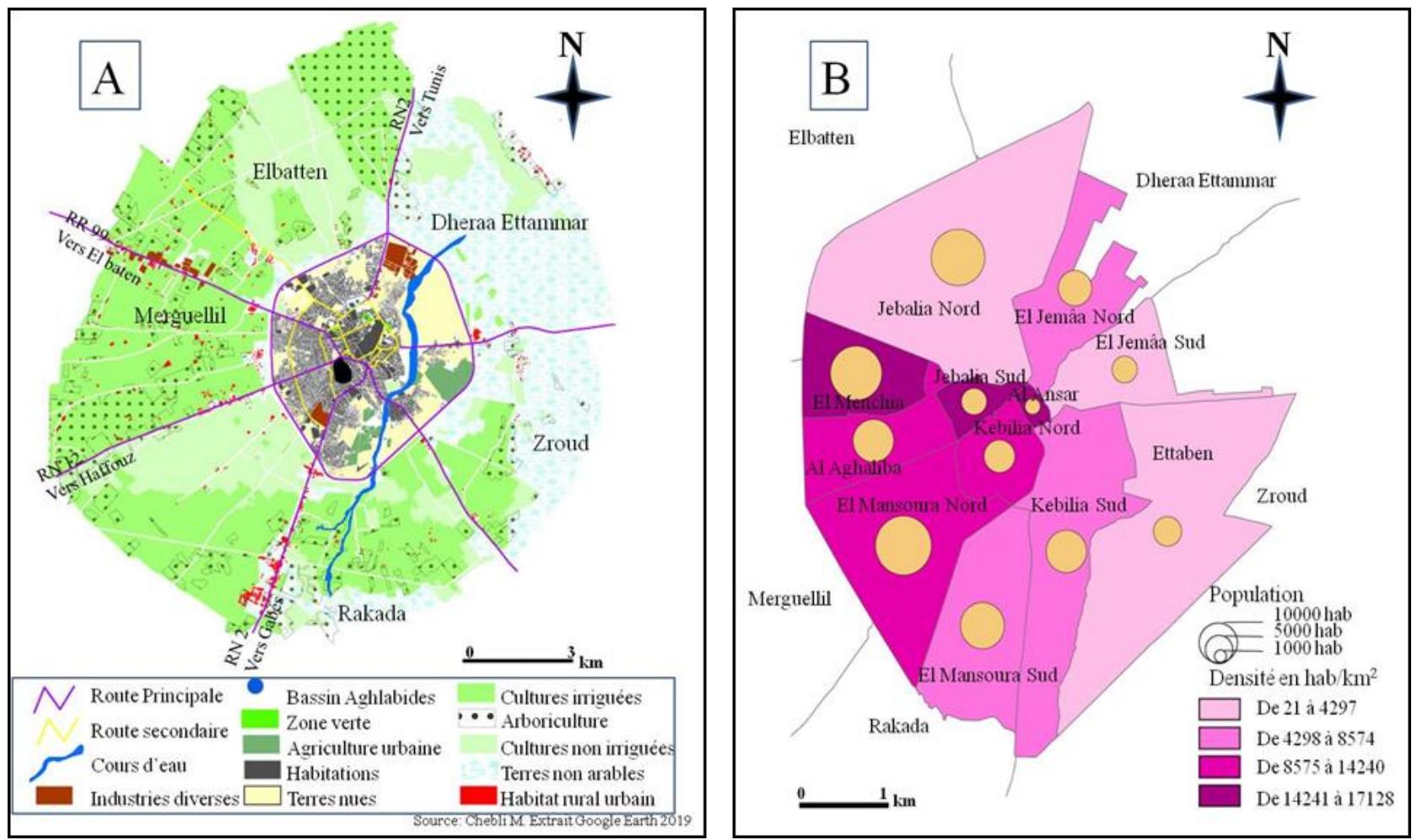

Figure 1. L'agglomération de Kairouan et ses alentours : occupation du sol (A) et population (B) ; source : Google Earth pour A et Institut National des statistiques pour B. The Kairouan agglomeration and its surroundings: soil occupancy (A) and population (B); data from Google Earth for $A$ and National Institute of Statistics for $B$.

Le climat de la zone d'étude est de type méditerranéen à nuance semi-aride, BSh selon la classification de Köppen et Geiger (climat de steppe chaud et sec). Il est caractérisé par une importante variabilité pluviométrique et une forte chaleur estivale (figure 3). La pluviométrie est de l'ordre de $300 \mathrm{~mm}$ en moyenne annuelle (Hénia, 2008). Les pluies d'hiver sont moins intenses que les pluies automnales. Des orages violents sont fréquents en septembre et octobre avec des précipitations qui peuvent atteindre 100 à 200 mm/24h entraînant souvent des inondations (Hénia, 2008). Durant la période 1973-2019, la station météorologique de Kairouan a enregistré une température maximale moyenne de $26,6^{\circ} \mathrm{C}$, et une minimale de $14,3^{\circ} \mathrm{C}$. L'analyse de la tendance des températures moyennes annuelles met en évidence une augmentation significative des maxima et des minimas avec des valeurs respectives de 0,5 et $0,7^{\circ} \mathrm{C} /$ décennie (figures $3 \mathrm{C}$ et $3 \mathrm{D}$ ). La moyenne de la température estivale (juin, juillet, août) varie entre 26,7 et $30^{\circ} \mathrm{C}$ avec une tendance significative à la hausse, notamment au niveau des minima qui est de l'ordre de $0,8^{\circ} \mathrm{C} /$ décennie. En été, les vents faibles à modérés prédominent : le jour, le vent synoptique venant de l'est est dominant alors que les nuits sont marquées par l'écoulement d'une brise de vallée canalisée par le relief, venant du nord à une vitesse moyenne faible, oscillant entre 1 et 4 $\mathrm{m} / \mathrm{s}$ (figure $3 \mathrm{~B}$ ).

\section{Données et méthodes}

L'étude de la répartition spatio-temporelle de l'ICU et du confort thermique est fondée sur les données météorologiques recueillies par des stations de mesures fixes et des campagnes de mesures itinérantes. 
Figure 2. La topographie de l'agglomération de Kairouan (source des données: SRTM). The topography of Kairouan agglomeration (data from SRTM).
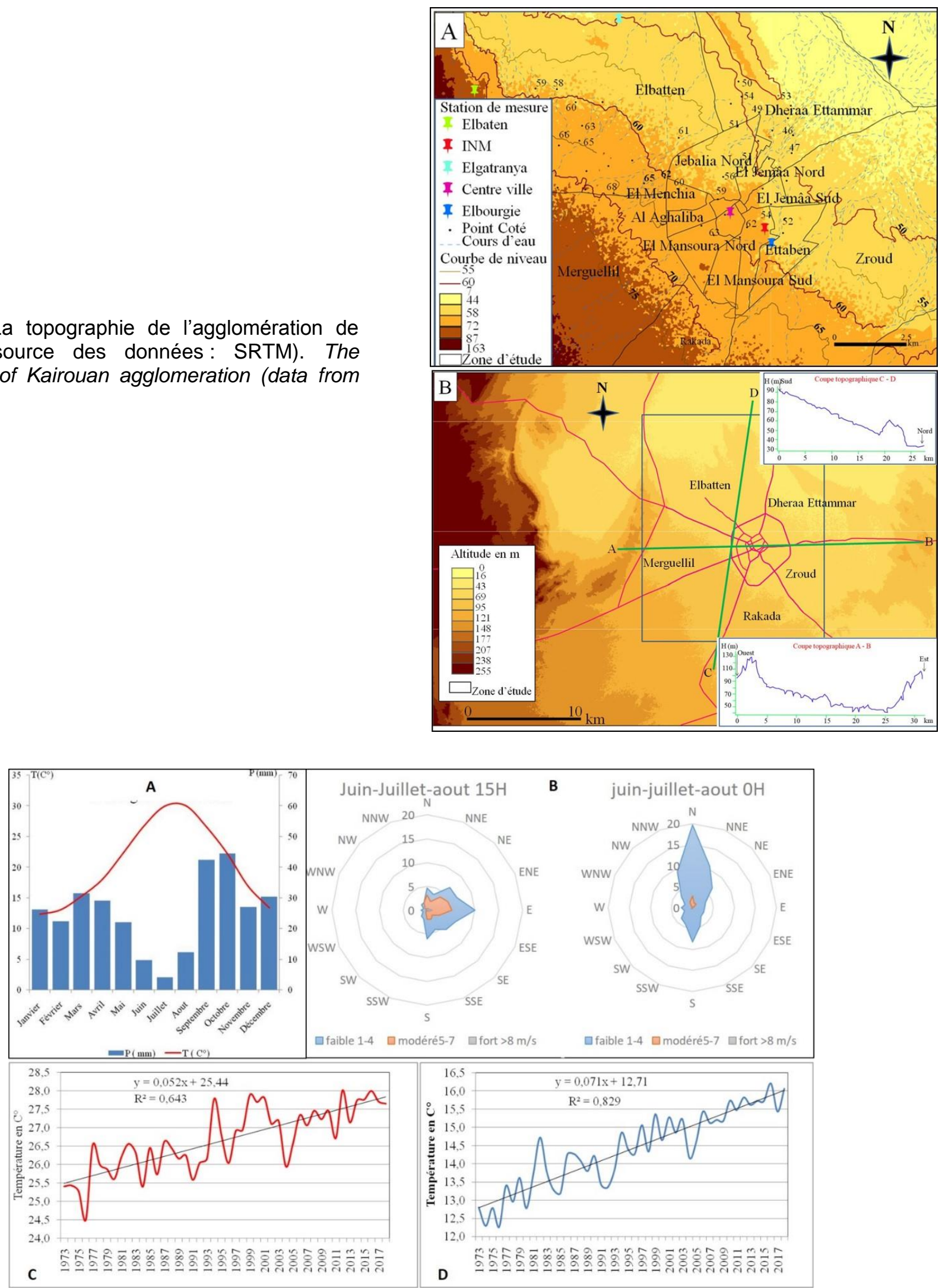

Figure 3. Diagramme ombrothermique $(A)$, rose moyenne du vent l'été à $0 \mathrm{~h}$ et $15 \mathrm{~h}(\mathrm{~B})$, tendance des températures maximales (C) et minimales (D) à Kairouan de 1973 à 2019 (données: Institut National de la Météorologie). Ombrothermal diagram (A), average windmill in summer at midnight and 3 p.m. (B), maximum (C) and minimum (D) temperatures trend in Kairouan from 1973 to 2019 (data from National Institute of Meteorology). 


\subsection{Les stations de mesures fixes}

Les stations automatiques mesurent différents paramètres météorologiques : température de l'air, humidité relative, vitesse et direction du vent et pression atmosphérique. Quatre stations ont été installées pour pallier le manque de données à l'échelle de l'agglomération, fournies uniquement par la station de l'Institut National de la Météorologie (INM), installée depuis 1923 (Bousnina, 2000). Trois stations de type Davis et une de type Campbell ont été installées depuis le
08/06/2019 sur les toits de bâtiments à une hauteur de $10 \mathrm{~m}$ (tableau 1). Cette hauteur s'est imposée pour protéger les stations de tout acte de vandalisme d'une part, et éviter l'effet de site et du trafic routier en garantissant une exposition au soleil pendant toute la journée, d'autre part. Les cinq stations, bien étalonnées, possèdent des sites urbains, périurbains et ruraux (figure 2). Les données issues des stations fixes sont précieuses car elles sont continues dans le temps mais demeurent ponctuelles dans l'espace, d'où le recours aux mesures itinérantes balayant toute l'agglomération.

Tableau 1. Quelques caractéristiques des stations météorologiques utilisées. Some characteristics of the meteorological stations used.

\begin{tabular}{|c|c|c|c|c|c|c|c|}
\hline $\begin{array}{c}\text { Nom de la } \\
\text { station }\end{array}$ & Site & $\begin{array}{c}\text { Type de } \\
\text { station }\end{array}$ & Latitude & Longitude & $\begin{array}{c}\text { Altitude } \\
\mathbf{( m )}\end{array}$ & $\begin{array}{c}\% \text { de } \\
\text { données } \\
\text { mesurées }\end{array}$ & $\begin{array}{c}\text { Périodes des } \\
\text { mesures }\end{array}$ \\
\hline EL Hosari & Centre- ville & Davis & $35^{\circ} 40$ & $10^{\circ} 5$ & 67 & 52,1 & $\begin{array}{c}10-24 / 06 / 2019 \\
02-28 / 08 / 2019 \\
01-31 / 10 / 2019\end{array}$ \\
\hline El bourgie & Périurbain & Davis & $35^{\circ} 39$ & $10^{\circ} 6$ & 58 & 60 & $\begin{array}{c}10 / 06 \text { au } 28 / 08 / 2019 \\
28-31 / 10 / 2019\end{array}$ \\
\hline Elgatranya & Campagne & Campbell & $35^{\circ} 44$ & $10^{\circ} 3$ & 50 & 100 & $14 / 06$ au 31/10/2019 \\
\hline INM & Centre- ville & $/$ & $35^{\circ} 40$ & $10^{\circ} 6$ & 63 & 100 & Depuis 1923 \\
\hline El baten & campagne & Davis & $35^{\circ} 42$ & $99^{\circ} 59$ & 78 & 84,2 & $\begin{array}{c}03 / 06 \text { au } 02 / 08 / 2019 \\
28 / 08 \text { au } 31 / 10 / 2019\end{array}$ \\
\hline
\end{tabular}

\subsection{Les mesures itinérantes}

Pour identifier le comportement spatial de la température dans l'agglomération de Kairouan à maille fine, nous avons eu recours à des campagnes de mesures itinérantes. Cette technique permet de cerner les facteurs susceptibles de générer des variations thermiques au sein de l'agglomération. Le but est d'augmenter la résolution spatiotemporelle des données thermiques. Les mesures ont été réalisées au milieu de la nuit, 5 à 6 heures après le crépuscule lorsque le rayonnement tellurique infrarouge atteint son paroxysme. Les points de mesure choisis sont représentatifs de la variété du tissu urbain et de la topographie. Nous avons utilisé deux thermomètres Testo 610 et 625 à temps de réponse rapide, à marge d'erreur inférieure à $0,5^{\circ} \mathrm{C}$ et d'une sensibilité très comparable. Les campagnes de mesure se sont déroulées en voiture roulant à une vitesse constante de $40 \mathrm{~km} / \mathrm{h}$ de façon que les sondes thermiques soient ventilées. Les capteurs sont tenus de façon qu'ils soient à l'abri de la chaleur dégagée par le moteur et la tôle de la carrosserie, ainsi que de l'air qui circule à l'intérieur des véhicules. Nous avons choisi des points facilement accessibles en voiture pour que la variation de la température mesurée entre les points soit due à une variation spatiale, et non à une variation temporelle (Charfi, 2012). Pour atteindre notre objectif, nous avons effectué vingt trajets centre-ville/campagne durant la saison chaude (juin à octobre), sur une distance de $7 \mathrm{~km}$ environ (tableau 2).

\section{Variabilité spatio-temporelle du champ thermique urbain à Kairouan}

\subsection{Evolution des températures horaires en été : cas de la période 03-27 août 2019}

L'analyse des données des postes météorologiques fixes montre un ICU d'une faible intensité à Kairouan, soit $2,5^{\circ} \mathrm{C}$ seulement, toutes situations météorologiques confondues (figure 4). 
Tableau 2. Quelques dates et heures des mesures itinérantes retenues à Kairouan pendant la saison chaude. Some dates and times of car survey retained in Kairouan during the hot season.

\begin{tabular}{|ccccc|}
\hline Date des mesures & Nb de transects & Nb $\mathbf{~ k m}$ & Heure & Type de temps \\
\hline $\mathbf{0 8 - 0 9 / 0 6 / 2 0 1 9}$ & 7 & 46,2 & $23 \mathrm{~h} 45-00 \mathrm{~h} 45$ & Temps calme \\
\hline $\mathbf{2 3 - 2 4 / 0 6 / 2 0 1 9}$ & 5 & 34,8 & $23 \mathrm{~h} 45-00 \mathrm{~h} 45$ & Temps calme \\
\hline $\mathbf{2 8 - 2 9 / 0 8 / 2 0 1 9}$ & 4 & 27,3 & $23 \mathrm{~h} 45-00 \mathrm{~h} 45$ & Temps perturbé \\
\hline $\mathbf{2 9 - 3 0 / 1 0 / 2 0 1 9}$ & 4 & 27,1 & $23 \mathrm{~h} 45-00 \mathrm{~h} 45$ & Temps calme \\
\hline
\end{tabular}

Par temps radiatif (vent faible et nébulosité de 0 octa), l'écart thermique ville-campagne atteint son maximum à la fin de la nuit en dépassant $3,1^{\circ} \mathrm{C}$ en moyenne. La concomitance de nombreux facteurs peut expliquer l'ICU dans l'agglomération de Kairouan : la végétation quasiment absente dans la zone urbaine, donc associée à une faible évapotranspiration, favorise une consommation de l'énergie principalement sous forme de flux de chaleur sensible. De même, le rayonnement des murs, des toits des bâtiments et des routes autour de la station urbaine, pendant la nuit, est déterminant (Nunez et Oke, 1976). En outre, la position de Kairouan comme ville carrefour au centre tunisien, explique la production assez importante de la chaleur anthropique dégagée par le trafic routier. C'est pour ces raisons que la station urbaine du centre-ville reste plus chaude toute la nuit.

Cependant, les températures baissent davantage dans les zones rurales par refroidissement radiatif. En effet, la végétation plus abondante et l'arrosage des périmètres irrigués augmentent la restitution de l'énergie sous forme de flux de chaleur latente. Enfin, il faut noter que l'intensité relativement modeste de l'ICU nocturne pourrait s'expliquer entre autres par l'emplacement des capteurs audessus des toits, à une hauteur de 6 à $8 \mathrm{~m}$, d'où une sous-estimation des températures en ville. Les toits choisis ne sont pas réfléchissants afin d'éviter une surestimation des températures le jour. Toutefois, en ville, avec la difficulté de trouver un site à l'abri de l'ombre et éloigné des parois, les toits des bâtiments restent un choix acceptable (Météo France, 2011). En effet, dans les rues, à cause de l'effet de parois, la température est plus élevée que sur le toit.

Le jour, par temps radiatif, l'écart thermique ville-campagne est très faible ou négatif, variant entre $0,3 \mathrm{C}^{\circ}$ et $-0,6^{\circ} \mathrm{C}$. La ville se transforme parfois en îlot de fraîcheur : l'absence d'un surcroît thermique urbain diurne pourrait s'expliquer d'une part par l'effet de l'ombre et la couleur blanche des toitures en ville, et d'autre part par le réchauffement plus rapide en zone rurale, dominée par des sols rendzines de couleur brun rougeâtre (Barbery et Mohdi, 1987). Néanmoins, par temps perturbé (vent fort et ciel couvert), l'ICU s'amenuise ou disparait. En effet, le vent impose le brassage de l'air et amenuise donc les écarts thermiques, mais ce type de situation reste rare à Kairouan en été (moins de 5 $\%)$.

A l'échelle de la journée, le site de la station du centre-ville est, en moyenne, plus chaud que celui de la station rurale d'Elgatranya, de $0,7^{\circ} \mathrm{C}$ (figure $4 \mathrm{C}$ ). Au début du jour, une à deux heures après le lever du soleil, les températures sont comparables dans les deux stations jusqu'au coucher du soleil. Au début de la nuit, le refroidissement nocturne est plus rapide dans la station rurale d'Elgatranya et moins marqué pour la station du centre-ville. Une ambiance chaude continue s'observe au centreville, expliquée par la restitution de la chaleur stockée par les bâtiments pendant le jour. En outre, la consommation de la chaleur sous forme latente est plus marquée à Elgatranya, malgré la sécheresse estivale, grâce à l'arrosage régulier des cultures maraîchères, très abondantes vers le site de mesure (figure 4). Les écarts se confirment davantage enfin de nuit par refroidissement radiatif plus marqué en zone rurale. D'où l'écart thermique atteint de $3,5^{\circ} \mathrm{C}$ en moyenne.

\subsection{Champs thermique urbain à Kairouan à partir des mesures itinérantes pendant la saison chaude}

Par ciel clair et vent faible, les mesures montrent un ICU intense avec un maximum de $9,9 \mathrm{C}^{\circ}$ (le 2324/06/2019). L'écart diminue par temps perturbé et ne dépasse pas $2,6^{\circ} \mathrm{C}$ (le 28-29/08/2019). 


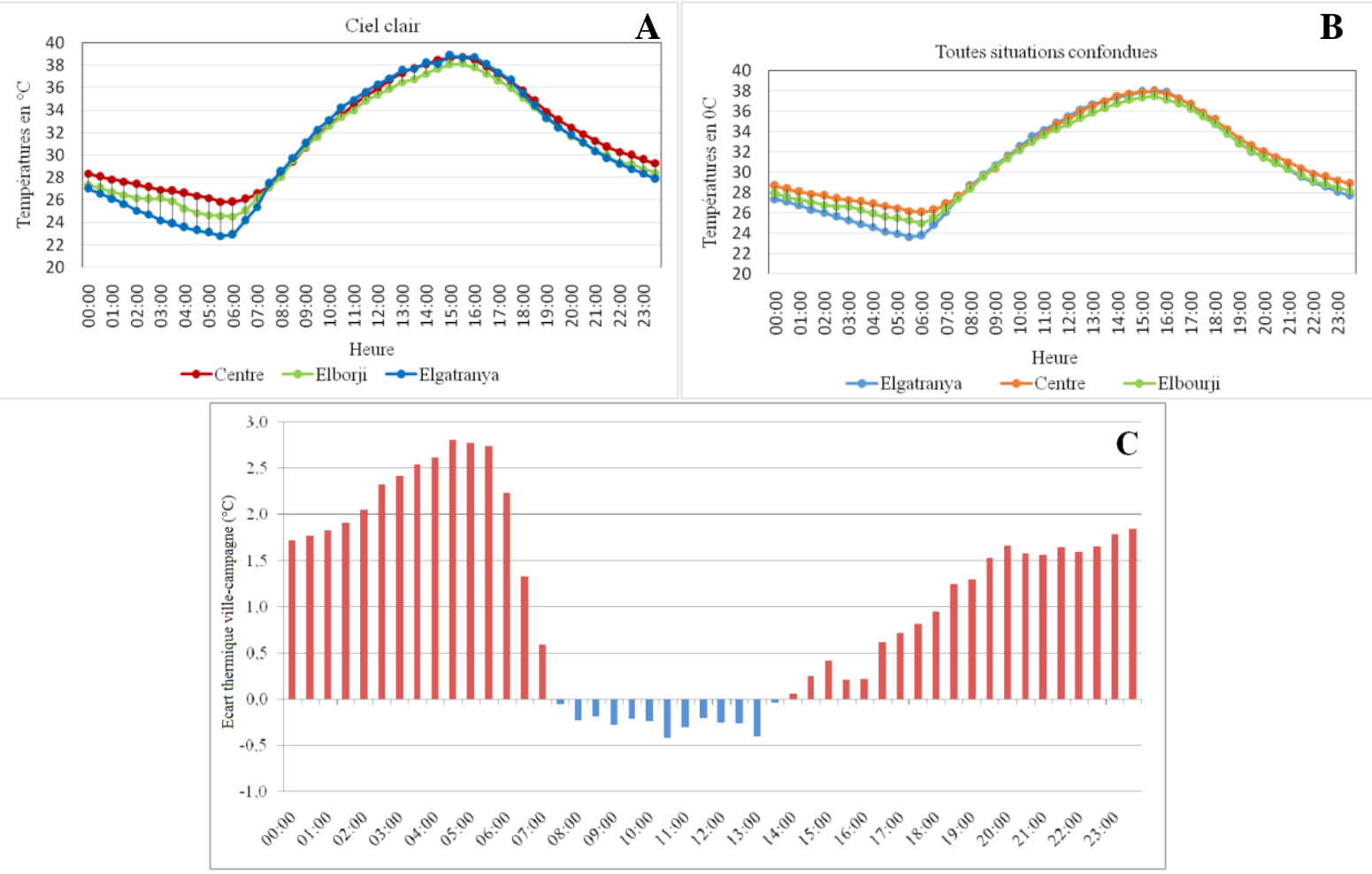

Figure 4. Evolution des températures estivales aux stations automatiques du centre-ville (urbaine), de Elbourji (périurbaine) et d'Elgatranya (rurale) du 3 au 27 août 2019 par temps radiatif (A) et toutes situations confondues (B), avec les écarts thermiques entre la station du centre-ville et la station rurale (C) ; données Davis, pas de temps de 30 min. Evolution of summer temperatures in the automatic stations of the town center (urban), Elbourji (peri-urban) and Elgatranya (rural) from 3 to 27 August 2019 in radiant weather $(A)$ and all situations combined (B), with thermal difference between the city centre station and the rural station (C); Davis data, 30min time interval.

\section{- Cas du 08-09/06/2019 à minuit}

Cette date correspond à un temps radiatif associé à des coups de sirocco. Cette campagne de mesure a duré 75 min et comporte plus de 100 points de mesures assez bien répartis dans l'espace, et couvrent une bonne partie de l'agglomération (figure 5A). Pendant cette nuit, l'ICU est intense. Nous avons enregistré un écart maximal entre les points E1 et $\mathrm{A} 18$ de $8,1^{\circ} \mathrm{C}$. En effet, la température de l'air augmente dans les zones densément urbanisées et peuplées (A1, G1, E1, E2, E4, F1, F2). Dans cette partie de la ville, les canyons urbains sont dépourvus de végétation et les matériaux de construction sont constitués généralement de brique rouge, à forte inertie thermique. La partie centrale de l'agglomération est donc un endroit de restitution importante de chaleur en phase nocturne. Un léger rafraichissant, ne dépassant pas $0,5^{\circ} \mathrm{C}$, est relevé près des bassins Aghlabides (C1) remplis d'eau, parce que les eaux stagnantes sont chaudes la nuit.

Sur les axes routiers, lorsqu'on s'éloigne de la ville, la température diminue à proximité des zones irriguées A17, A18, A19, B11, B9, G20 et F15 qui correspondent à des îlots de fraîcheur grâce à l'effet de la végétation, à l'arrosage et à la brise de l'oued (Merguellil et Zroud) qui achemine l'air frais de l'intérieur du continent, comme c'est le cas aux points de mesure D8, E3 et E6. En milieu rural, et à distance égale du centre-ville (E1), les périmètres irrigués sont $3^{\circ} \mathrm{C}$ plus frais que les terres labourées nues, comme c'est le cas du gradient thermique entre les points de mesure A16 et A18 situés à $7 \mathrm{~km}$ du centre-ville (figure 5A).

\section{- Cas du 23-24/06/2019 à 23h30}

La figure $5 \mathrm{~B}$ montre un ICU nocturne intense, comparable à celui identifié dans des villes de taille supérieure, de l'ordre de $9,9^{\circ} \mathrm{C}$. Le noyau le plus chaud occupe la zone centrale densément urbanisée (à forte inertie thermique), avec des prolongements au nord, à l'ouest et au sud, vers des axes routiers plus denses. Jusqu'à un rayon d'environ $3 \mathrm{~km} \mathrm{du}$ centre-ville, les écarts restent inférieurs à $2^{\circ} \mathrm{C}$, ce 
qui s'explique vraisemblablement par l'homogénéité du tissu urbain dominé par les structures horizontales et par l'absence de végétation. A proximité du centre-ville, le passage par un canyon traversant la Medina s'accompagne par une baisse relative de la température de l'ordre de $1^{\circ} \mathrm{C}\left(31,1^{\circ} \mathrm{C}\right.$ à D3 et $29,6^{\circ} \mathrm{C}$ à $\left.\mathrm{D} 2\right)$, ce qui s'explique vraisemblablement par la présence des plantes ligneuses et la faible capacité de réchauffement des pierres taillées.

Dès que nous nous éloignons du centre-ville, le tissu urbain devient lâche et les températures baissent d'une manière parfois brutale car l'agglomération de Kairouan est entourée par une ceinture de cultures irriguées (figure 5B). Vers le nord-ouest par exemple, la température baisse de $5^{\circ} \mathrm{C}$ entre les points $\mathrm{A} 2\left(29,9^{\circ} \mathrm{C}\right)$ et $\mathrm{A} 4\left(24,9^{\circ} \mathrm{C}\right)$ distants d'un kilomètre environ. Le refroidissement marqué au niveau du point A4 s'explique conjointement par la présence des périmètres irrigués et l'encaissement du site traversé par un petit ravin. Vers le nord, une baisse de $2,2^{\circ} \mathrm{C}$ a été observée dès qu'on s'éloigne de la zone urbaine entre $\mathrm{C} 3$ et $\mathrm{C} 4$, due au changement brusque de la nature d'occupation du sol (figure 5B). En dehors de la zone urbaine, l'occupation du sol et la position géographique deviennent des paramètres déterminants de la variation spatiale de la température. En effet, le transect A montre que le passage par les périmètres irrigués d'El Baten (A4, A5 et A11) à l'ouest s'accompagne par une baisse de plus de $2 \mathrm{C}^{\circ}$ par rapport au noyau urbain d'El Baten et sa zone industrielle (A8 et A9). De même, en passant par la piste agricole d'Elgatranya, nous avons enregistré une baisse de $1,8^{\circ} \mathrm{C}$ au niveau des points B10, B11et B12 situés dans la zone irriguée par rapport à la zone peu urbanisée (B7, B9). Le même phénomène a été observé au sud entre E5 et E6 avec un écart de $2,5^{\circ} \mathrm{C}$. Un air frais canalisé par l'oued Marguellil est enregistré sur la route de Rakada au sud (E11). Il s'agit des coulées fraîches (brise de vallée) provenant de l'intérieur du continent à une vitesse avoisinant $4 \mathrm{~m} / \mathrm{s}$ au maximum. Nous notons enfin que le surcroit thermique enregistré à B6 par rapport à son environnement immédiat s'explique par l'effet de l'incinération des déchets au moment des prises de mesure des températures.

\section{- Cas du 28-29/08/2019 à 23h30}

Pendant la journée, un vent fort a soufflé de l'est et le ciel a été voilé ( 6 octas). La nuit, le vent a été modéré venant du nord à une vitesse de $5 \mathrm{~m} / \mathrm{s}$ avec une forte nébulosité. La figure 5C montre un champ thermique plus ou moins homogène et un ICU relativement faible de l'ordre de $2,6^{\circ} \mathrm{C}$ seulement. Cependant, les variations thermiques restent très significatives. En effet, la partie centrale de l'agglomération n'est pas toujours la plus chaude. Les températures maximales, enregistrées à l'ouest de l'agglomération, sont de l'ordre de 24 à $25^{\circ} \mathrm{C}$. Le vent soufflant du nord réduit l'intensité de l'ICU par effet de brassage et pousse le panache urbain vers le sud et l'ouest. Cependant, la zone rurale reste la plus fraîche.

L'écart thermique intra-urbain est généralement faible de l'ordre de 1 à $2,5^{\circ} \mathrm{C}$ (figure 5D). Les valeurs maximales sont enregistrées par temps torride de sirocco $\left(7^{\circ} \mathrm{C}\right)$ alors que le gradient villecampagne ne dépasse pas $5^{\circ} \mathrm{C}$ durant le reste des jours radiatifs en été. Ces écarts s'amenuisent par temps perturbé propice au brassage de l'air pour ne pas dépasser $2^{\circ} \mathrm{C}$.

\section{- Cas du 29-30/10/2019 à 23h30}

La nuit du 29 octobre est caractérisée par un beau temps calme. Nous avons enregistré un ICU remarquable de $5,2^{\circ} \mathrm{C}$. Au niveau de la zone urbaine, l'écart thermique entre les quartiers densément peuplés est faible et ne dépasse pas $2^{\circ} \mathrm{C}$. Les points de mesure situés près des cours d'eau correspondent à des micro-îlots de fraîcheur comme c'est le cas au niveau de l'Oued Elmelah (C2, C3, $\mathrm{C} 4)$. Le même phénomène a été observé à proximité des petits parcs urbains (D12). En effet, le passage à côté du parc du Mausolée situé au nord-ouest de l'agglomération s'accompagne par une baisse des températures de $1,5^{\circ} \mathrm{C}$ environ.

Le point D12 situé au nord du parc est plus frais que B1 et D13 situés dans les quartiers voisins de part et d'autre du parc (figure 5D). Il s'agit vraisemblablement de l'effet de la végétation relativement dense et régulièrement arrosée qui modifie les éléments du bilan d'énergie en favorisant la consommation de l'énergie sous forme latente pour l'évaporation. 
Figure 5. Spatialisation de la température de l'air à Kairouan par type de temps : par temps radiatif, i.e. valeurs proches de la normale (A et $D)$, torride $(B)$, perturbé $(C)$ et synthèse de l'ICU par type de temps $(E)$; mesures itinérantes par capteurs Testo, fond à partir de Google Earth et Nasrallah (2019). Spatialization of the air temperature in Kairouan by the type of weather: by radiative weather (values close to normal, $A$ and $D$ ), torrid (B), disturbed (C) and $\mathrm{UCl}$ synthesis by weather type $(E)$; car survey with Testo sensors; background from Google Earth and Nasrallah (2019).
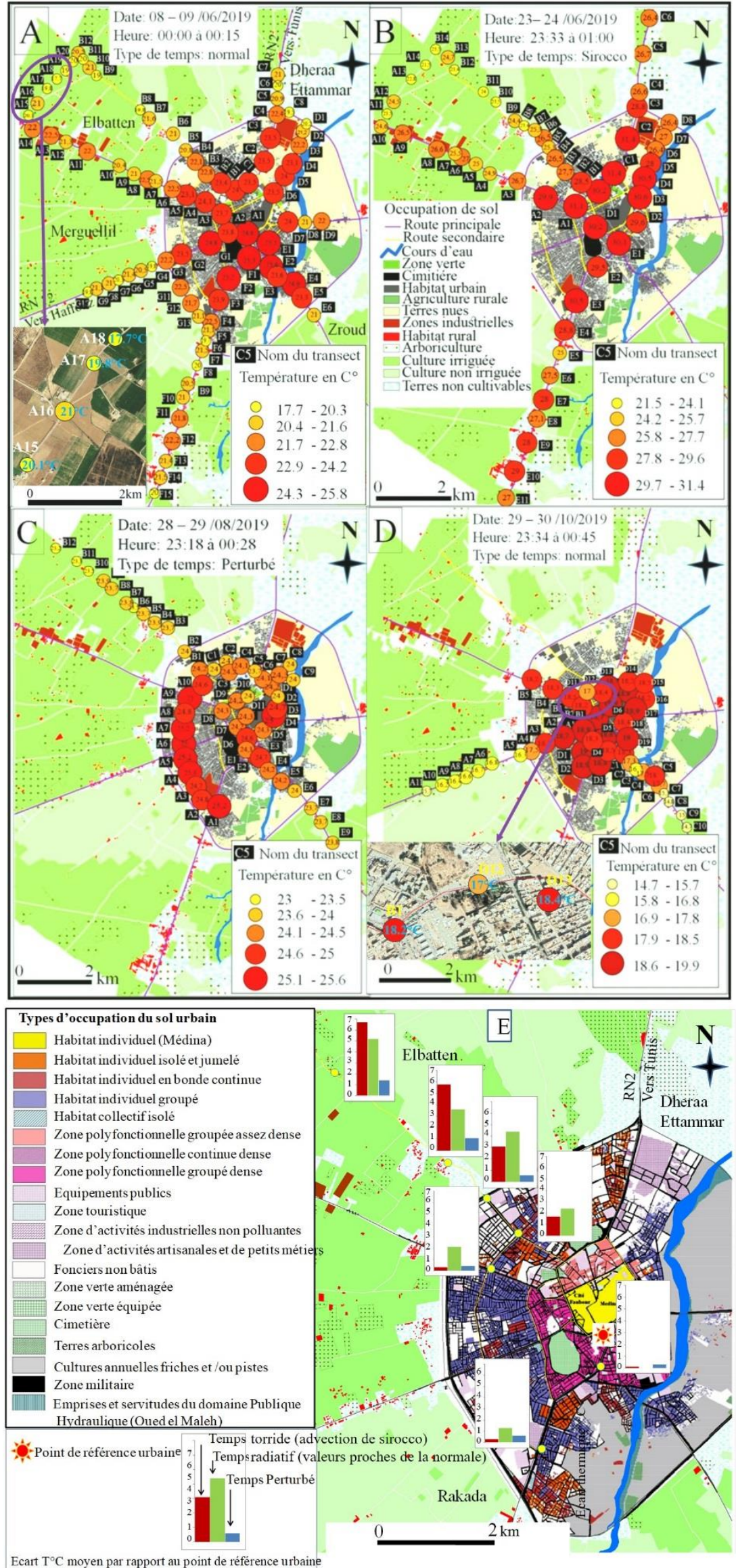

Page 9 de 15 
Dans ce jardin, la quantité de chaleur consacrée pour réchauffer l'air est moindre (Charfi, 2012). En dehors de l'agglomération, les températures baissent dans les zones rurales qui entourent la ville et varient entre 16,8 et $14,7^{\circ} \mathrm{C}$.

La figure $5 \mathrm{E}$ qui synthétise les moyennes des écarts thermiques par rapport au point de référence urbaine par type de temps sur la période étudiée, montre que 1'écart thermique intra-urbain est généralement faible de l'ordre de 1 à $2,5^{\circ} \mathrm{C}$ en fonction de la densité du tissu urbain. Les gradients les plus marqués sont repérés au nord de l'agglomération où s'étalent les quartiers à habitat pavillonnaire. Au sud, les écarts thermiques intraurbains se dégradent sensiblement à cause de l'étalement d'un tissu urbain dense.

Les valeurs maximales sont enregistrées par type de temps calme. Le gradient thermique centre/campagne atteint son paroxysme $\left(7\right.$ à $\left.9^{\circ} \mathrm{C}\right)$ par temps torride quand souffle le sirocco (vent chaud et sec). Ces écarts s'amenuisent $\left(2^{\circ} \mathrm{C}\right)$ par temps perturbé propice à l'égalisation des températures par effet de brassage.

\section{Confort thermique et îlot de chaleur dans l'agglomération de Kairouan}

\subsection{La variation du Heat Index (HI) et Temperature Humidity Index (THI) mesurés par les postes météorologiques fixes}

Le confort thermique, une sensation subjective, dépend de plusieurs paramètres météorologiques, (Epstein et Moran, 2006 ; Dahech, 2014). Pour exprimer la relation entre le confort thermique, exprimé par la température ressentie, et l'ICU dans la ville de Kairouan et sa périphérie, 35 jours ont été sélectionnés en été 2019 pour lesquels les moyennes des températures et de l'humidité sont complètes et coïncident avec une situation de temps radiatif calme. Deux indices de confort ont été retenus, le Heat Index (HI) et le Temperature Humidity Index (THI) :

- L'indice HI permet d'estimer les températures ressenties en tenant compte du taux d'humidité. "Il a été mis au point par le département américain de météorologie nationale afin de prévenir les décès et les accidents en cas de vague de chaleur en été. Cet indice est largement utilisé aujourd'hui (l'Institut National de Recherche et de Sécurité pour la prévention des accidents du travail - INRS, 2004). La formule de calcul du HI se base sur deux paramètres : la température de l'air $\left(T^{\circ} C\right)$ et l'humidité relative $R(\%)$. C'est le fruit d'un modèle statistique élaboré par Rothfusz (1990) et validé avec une marge d'erreur de $\pm 1,3^{\circ} \mathrm{C}$. Son calcul est encore plus simple moyennant le calculateur automatique du site web wpc.ncep.noaa.gov/html/heatindex.shtml " (Dahech, 2014).

- L'indice THI de Thom croise également la température avec l'humidité de l'air. La formule de cet indice est :

$$
\mathrm{THI}=\mathrm{T}-((0,55-0,0055 * \mathrm{H}) *(\mathrm{~T}-14,5))
$$

Avec $\mathrm{T}$, la température de l'air en ${ }^{\circ} \mathrm{C}$ et $\mathrm{H}$, l'humidité relative en \%. Les classes des ambiances biothermiques selon l'indice THI sont résumées dans le tableau 3.

Tableau 3. Les catégories de l'Indice de Thom (Thom, 1959). Categories of the Thom Index (Thom, 1959).

\begin{tabular}{|cc|}
\hline THI en $\mathrm{C}^{\circ}$ & Ambiance \\
\hline THI $\geq 30$ & Torride \\
\hline 29,9 à 26,5 & Très chaud \\
\hline 26,4 à 20 & Chaud \\
\hline 19,9 à 15 & Confortable \\
\hline 14,9 à 13 & Frais \\
\hline 12,9 à $-1,7$ & Froid \\
\hline$-1,8$ à $-9,9$ & Très froid \\
\hline THI $\leq-10$ & Extrêmement froid \\
\hline
\end{tabular}

Les avantages de ces deux indices sont leur facilité de calcul, leur applicabilité à des situations de chaleur en climat méditerranéen et le fait qu'ils utilisent deux paramètres climatiques communément mesurés par les stations météorologiques. En effet, le HI a été utilisé par Monteiro et al. (2013) pour Porto au Portugal et par Zoumakis et al. (2012) pour Thessaloniki en Grèce. L'indice de Thom a été utilisé par certains climatologues en Tunisie, comme par exemple Ben Boubaker (2010).

Certains indices intégrant d'autres paramètres complémentaires à la température et à l'humidité, 
comme le Temperature Humidity Wind Index (THW) et le Temperature-Humidity-Wind-Sun (THWS) (Steadman, 1979 et 1984) ne sont pas utilisés dans ce travail car une grande partie des travaux de terrain, à savoir les mesures itinérantes, est réalisée la nuit par temps calme donc en l'absence du vent et du rayonnement. Les résultats sont obtenus à l'échelle tri-horaire $(00,3,6,9,12$, 15,18 et $21 \mathrm{~h}$ ) et sont représentés dans la figure 6 .

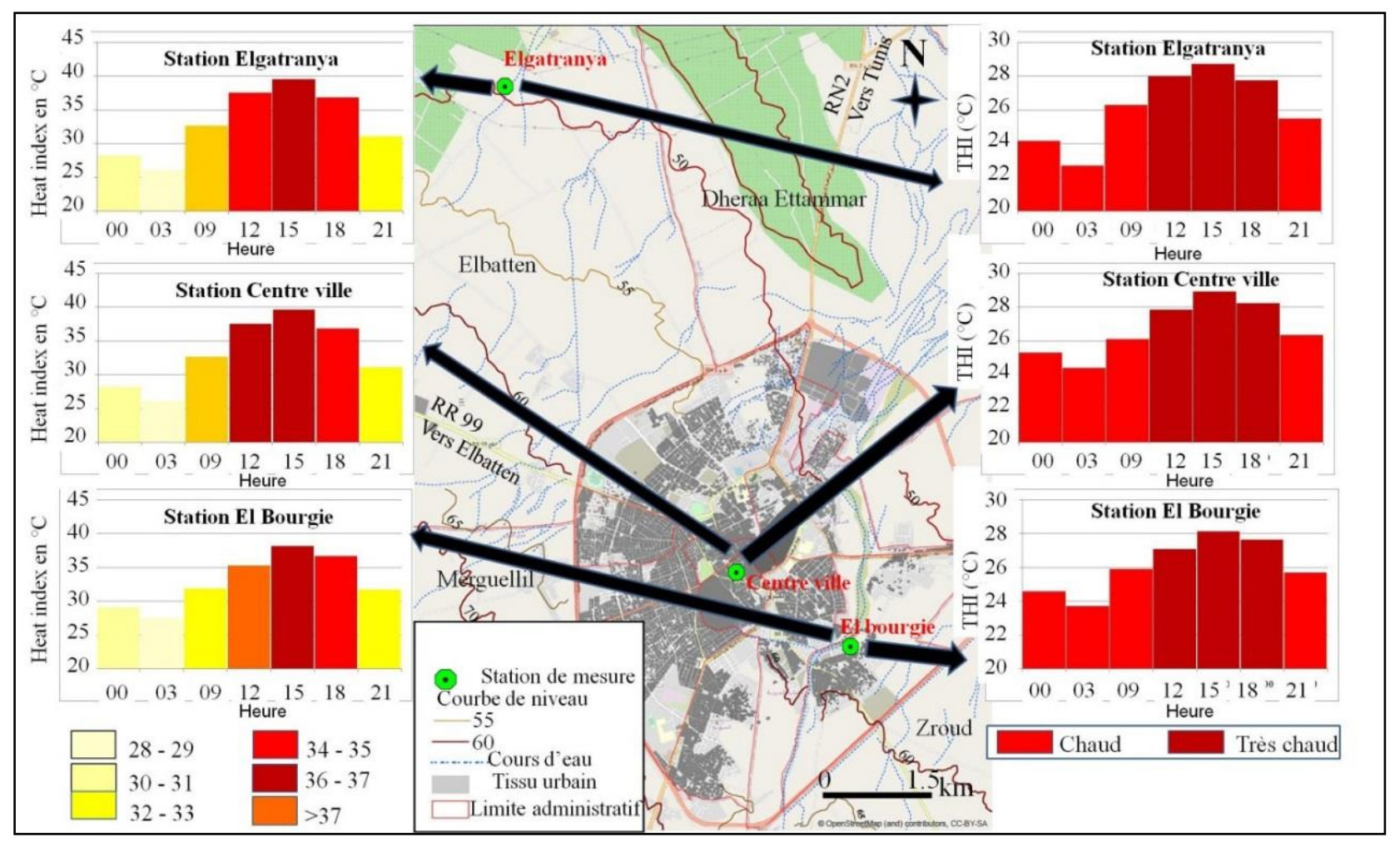

Figure 6. Répartition des indices de confort $\mathrm{HI}$ et THI moyens aux stations de mesures à $0,3,6,9,12,15,18$ et $21 \mathrm{~h}$, calculés sur la période du 2 au 28 août 2019 (données à partir de stations Davis). Distribution of the average comfort index $\mathrm{HI}$ and $\mathrm{THI}$ in the measurement stations at midnight, 3, 6 and $9 \mathrm{am}$, midday, 3, 6, and 9 pm, calculated from 2 to 28 August 2019 (from Davis data).

Les résultats obtenus par les trois stations montrent une situation d'inconfort thermique dans l'agglomération de Kairouan durant toute la journée selon les deux indices indiqués ci-dessus. Selon l'indice de Thom, un temps chaud à très chaud persiste toute la journée. De même, les valeurs de $\mathrm{HI}$ dépassent $28^{\circ} \mathrm{C}$ dans la plupart du temps (figure 6). La situation la plus inconfortable au cours de la journée a été observée entre $12 \mathrm{~h}$ et $18 \mathrm{~h}$. Cet inconfort s'explique par la forte chaleur estivale, par la durée d'ensoleillement assez longue et s'accentue par l'effet de l'ICU en ville. Ces conditions ont des répercussions nocives sur la santé humaine, comme le risque de coups de chaleur et d'accidents cardiovasculaires (Barnett, 2007). La situation s'améliore à partir de $21 \mathrm{~h}$ jusqu'à $9 \mathrm{~h}$ du matin. A minuit, les deux stations d'El Bourgie (périurbaine) et d'Elgatranya (rurale) enregistrent une légère amélioration du niveau de confort suite à la baisse de la température pour deux raisons : 1) la zone d'EL Bourgie est alimentée en air frais canalisé par les cours d'eaux des oueds El Maleh et Zroud au sud de la ville ; 2) la zone de la station d'El Gatranya est fortement irriguée, donc relativement fraîche et alimentée par la brise de vallée qui se déclenche à partir de minuit. Par contre, la situation d'inconfort persiste au centre-ville, la nuit, à cause de l'ICU.

\subsection{Les variations spatiales du Heat Index dans l'agglomération de Kairouan vers minuit, pendant la saison chaude}

La répartition spatiale du confort thermique est analysée à partir des campagnes de mesures itinérantes pendant la saison chaude (figure 7). 
- La nuit du 08-09/06/2019 : l'indice HI augmente dans la partie sud de l'agglomération (points F1, F2, E1, E2). Le seuil de prudence $27^{\circ} \mathrm{C}$ est atteint. Cette situation d'inconfort s'explique par la chaleur emmagasinée et restituée par les bâtiments et repoussée au sud par la brise de vallée venant du nord. Cependant, le reste de l'agglomération, notamment la partie septentrionale, est caractérisée par une condition de confort liée aux brises de campagne et ou brise de vallée, comme c'est le cas du point D8 situé dans la vallée de Zroud à l'est de l'agglomération (figure 7A). De même, les espaces verts se transforment en micro-îlots de confort qui contribuent à l'amélioration du niveau du $\mathrm{HI}$ en baissant la température, comme le Parc du Mausolée (A3) ou le $\mathrm{HI}$ ne dépasse pas $25^{\circ} \mathrm{C}$.

- La nuit du 23-24/06/2019 est marquée par une situation d'inconfort au centre de l'agglomération et vers les axes routiers. Le dépassement du seuil de prudence extrême $32^{\circ} \mathrm{C}$ est repéré dans une grande partie de l'agglomération (figure 7B). Le champ de confort est relativement hétérogène avec un écart $\mathrm{HI}$ de $6^{\circ} \mathrm{C}$ entre le centre-ville, très inconfortable, et le milieu rural environnant, relativement confortable Cette situation s'explique par la concomitance de deux facteurs: le surcroit thermique urbain expliqué par l'importance des surfaces imperméables à forte inertie thermique et les conditions météorologiques (coups de sirocco, advection d'air chaud et sec). En milieu rural, dans la partie nord-ouest (la zone d'El Baten et El Gatranya), l'indice $\mathrm{HI}$ varie entre 25 et $26^{\circ} \mathrm{C}$ : la situation relativement confortable s'explique par la brise de campagne (fraîche) et par l'importance des périmètres irrigués qui jouent un rôle rafraichissant. Pendant cette nuit, les espaces verts en milieu urbain ont contribué à la baisse de l'indice $\mathrm{HI}$ de $1^{\circ} \mathrm{C}$ au maximum, comme c'est le cas au niveau de D2.

- La nuit du 28-29/08/2019 : il s'agit d'une nuit nébuleuse avec un vent modéré. Pour cette raison, le champ de confort est homogène. L'écart maximal du $\mathrm{HI}$ entre le centre et la campagne est de l'ordre de $2{ }^{\circ} \mathrm{C}$ (figure $7 \mathrm{C}$ ). L'ambiance thermique se caractérise par une situation d'inconfort au centre de l'agglomération et surtout dans les quartiers densément peuplés comme El Menchia, Alaghliba, Mansoura Nord (A1, A10), où le seuil de prudence $27^{\circ} \mathrm{C}$ est atteint. Une baisse relative de $1^{\circ} \mathrm{C}$ seulement a été enregistrée au niveau des parcs urbains Sidi Saahbi (D2) et du Mausolée (D10). A l'extérieur de la zone urbaine, l'indice HI diminue et le niveau de confort s'améliore légèrement à cause de la baisse des températures.

Si nous comparons les températures ressenties exprimées par l'indice HI aux températures de l'air, le 9 juin 2019, soit une situation proche de la normale, à minuit, nous remarquons une détérioration du confort, comme le montre la figure 7D. Cette dernière laisse voir une faible différence entre les deux paramètres comparés au centre-ville car l'air y est relativement sec. Cependant, en zone rurale, dominée par les cultures irriguées, le taux d'humidité est relativement élevé et participe à une détérioration du confort thermique. En effet, à titre d'exemple, dans la zone d'Elgatranya, au nordouest, un écart positif de $7^{\circ} \mathrm{C}$ est observé entre le $\mathrm{HI}$ et la température de l'air à cause d'un taux d'humidité relative avoisinant $90 \%$.

\section{Discussion}

Cette étude en climatologie urbaine est originale car elle s'intéresse à un espace non étudié et compare l'ICU à l'îlot de confort thermique. L'ensemble des données issues des mesures fixes et itinérantes effectuées durant la saison chaude nous a permis de cerner les résultats suivants :

- l'îlot de chaleur urbain identifié par les postes fixes est important mais il dépend de plusieurs paramètres: le type de temps, la période de la journée et la saison, l'environnement immédiat du site de mesure et le site. L'ICU s'avère légèrement moins intense en moyenne que celui mesuré dans des villes de taille supérieure comme Tunis (2,8 millions d'habitant) et Sfax (600 000 habitants).

Toutefois, certaines situations météorologiques sont propices à un ICU intense $\left(9^{\circ} \mathrm{C}\right)$ qui s'explique par l'effet de la topographie et de l'irrigation qui creusent les écarts thermiques. 


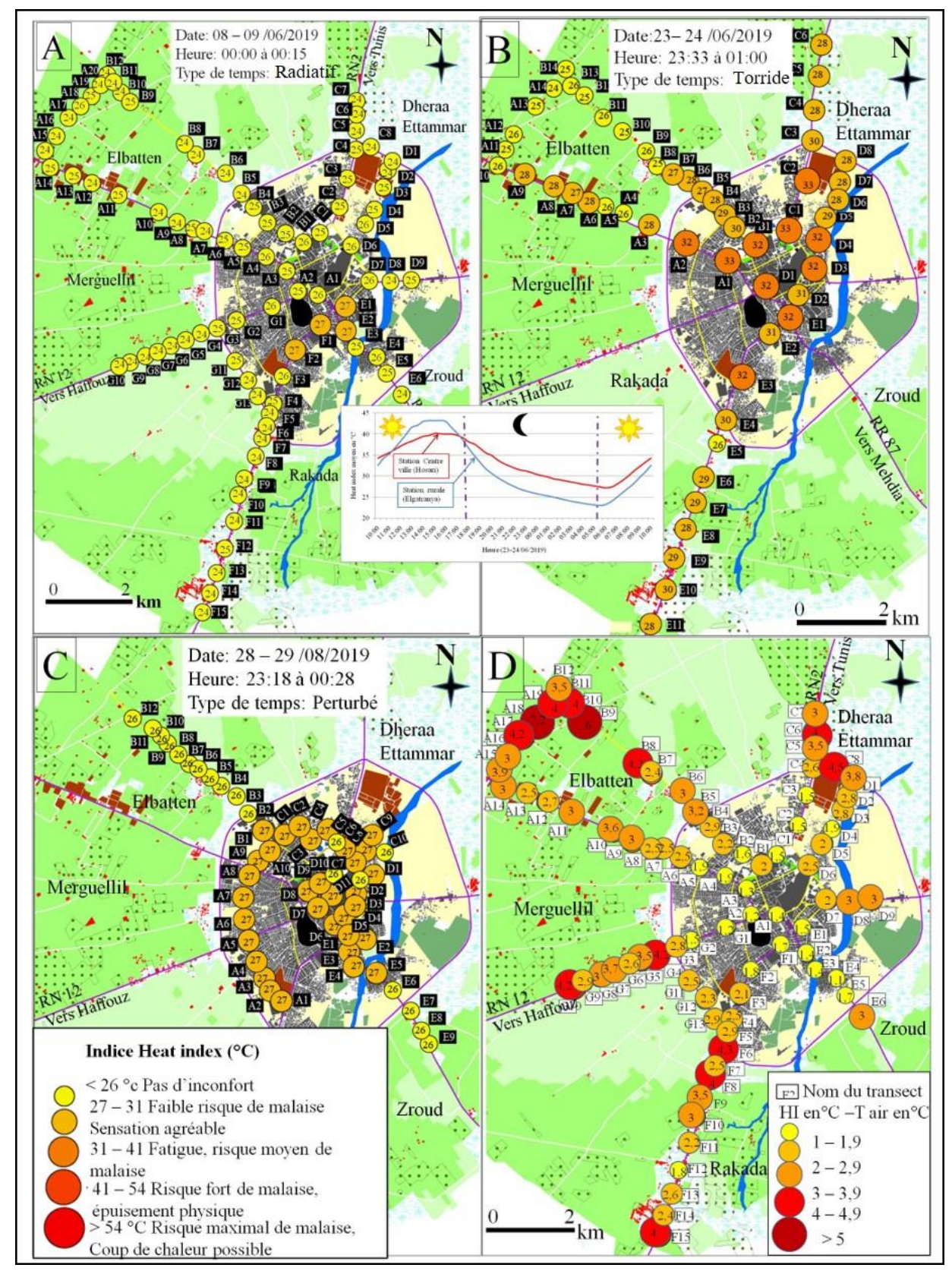

Figure 7. Spatialisation du HI à Kairouan par type de temps pendant trois dates : par temps radiatif avec des valeurs proches de la normale $(A)$, torride $(B)$, perturbé $(C)$, ainsi que l'écart entre $\mathrm{HI}$ et la température de l'air la nuit radiative du 8-9 juin 2019 (D). Spatialization of Heat index in Kairouan by type of weather during three dates: in radiative weather (values close to normal) $(A)$, torrid $(B)$, disturbed $(C)$ and difference between $\mathrm{HI}$ and air temperature at the night of June 8-9, 2019 (D).

A l'échelle fine, les campagnes de mesures ont montré que le tissu urbain compact et quasihomogène en termes de densité et volume du bâti engendre de très faibles variations des températures dans la partie dense de l'agglomération. Toutefois, les quartiers densément peuplés comme El Menchia ou la Cité Med-Ali sont les plus chauds. Ceci s'explique par la morphologie urbaine, d'une part, et par les matériaux de construction à forte inertie thermique, d'autre part.

- les résultats fournis après le calcul du HI montre que le degré de confort varie à l'échelle de l'agglomération et ses environs, et durant la journée. Les situations les plus inconfortables s'observent entre midi et $18 \mathrm{~h}$. L'inconfort s'explique par la forte chaleur estivale (Dahech, 
2014), notamment quand le vent souffle du secteur méridional. Cette situation s'améliore vers la fin de la journée à partir de minuit pour les postes météorologiques ruraux et périurbains, et à partir de $3 \mathrm{~h}$ du matin pour le centre-ville. Les campagnes de mesures itinérantes durant la saison chaude montrent que l'ambiance climatique dans le centreville est légèrement inconfortable par rapport à la périphérie et à la campagne, avec un écart au niveau du HI plus bas que l'ICU. En milieu rural, la sensation de confort baisse à cause d'un taux d'humidité élevé.

Deux indices de confort sont utilisés dans ce travail : HI et THI. Le premier semble plus adapté à la zone d'étude car il reflète mieux la sensation de chaleur à l'échelle de l'agglomération. Ce constat est validé par des travaux d'enquête réalisés durant l'été 2020. De même, les campagnes de mesures réalisées instantanément par deux équipes pendant l'été 2020 confirment les résultats obtenus en 2019. Il sera judicieux dans les prochaines études d'intégrer davantage le vent comme paramètre expliquant la variation spatiotemporelle des températures et du confort thermique.

\section{Conclusion}

Les données recueillies durant la saison chaude 2019 à travers les postes fixes et les mesures itinérantes de la température de l'air étaient extrêmement riches. Elles nous ont permis une meilleure connaissance du champ thermique dans l'agglomération de Kairouan. Le champ thermique urbain est peu variable à cause de l'homogénéité du tissu urbain dominé par des bâtiments ne dépassant pas trois étages. Ici, comme partout dans le monde, les écarts thermiques ville-campagne s'expliquent par la nature du substrat et un décalage dans le régime journalier de la température, creusant davantage les écarts thermiques instantanés. A Kairouan, l'ICU s'accentue par le phénomène de la brise de vallée, venant du nord, très fréquente dans la région ainsi que par l'étalement des périmètres irrigués, en périphérie. Ces derniers se transforment la nuit en îlots de fraîcheur et permettent de creuser davantage l'écart thermique d'au moins $3^{\circ} \mathrm{C}$ par rapport au centre-ville. De ce fait, la végétalisation des boulevards par des espèces adaptées au climat local comme l'eucalyptus, répandu dans la région, ainsi que les parcs urbains arrosés pourraient être une solution pour réduire l'ICU. Ces solutions produisent l'ombre et la fraîcheur, à condition de prendre en considération le problème de la pénurie d'eau. L'ICU peut aggraver certaines situations météorologiques très inconfortables, en l'occurrence en cas d'advection du sirocco, et amplifie, par conséquence, le besoin en énergie électrique utilisée pour la climatisation. Il est également probable que les cas de morbidité respiratoire et cardiovasculaire augmentent par temps caniculaire surtout dans les quartiers densément peuplés et habités par une population vulnérable. Des enquêtes réalisées pendant l'été 2020 pour cerner le degré de vulnérabilité et la résilience de la population face à la forte chaleur dans les quartiers denses de la ville, ont montré plusieurs modes d'adaptation à la forte chaleur, notamment en phase nocturne, comme l'utilisation des toits des maisons comme dortoirs.

Remerciements : les auteurs adressent leurs vifs remerciements à Mohamed Feki et Marwen Ghribi qui ont participé aux mesures itinérantes. Les travaux de terrains ont été financés en partie par le projet PHC Maghreb "Îlots de chaleur urbains dans les villes du sud de la Méditerranée : diagnostic et résilience" (39361WA).

\section{Références}

Barbery J. \& Mohdi M., 1987. Carte des ressources en sols de la Tunisie, Feuille de Kairouan. Ministère de l'Agriculture, Direction des sols, Tunisie, $49 \mathrm{p}$.

Barnett A. G., 2007. La température et les décès cardiovasculaires chez les personnes âgées aux ÉtatsUnis. Epidemiology, 18 (3), 369-372.

Beltando G., 2011. Les climats: processus, variabilité et risques. A. Colin, $286 \mathrm{p}$.

Ben Boubaker H., 2010. Les paroxysmes climato-thermiques en Tunisie: approches méthodologiques et études de cas. Climatologie, 7, 57-87.

Bousnina A., 2000. Variations et variabilité des températures en Tunisie 1901-1985, Tome 1. Publication de la faculté des Sciences Humaines et Sociales de Tunis, 498 p.

Carrega P., 2013. Le climat urbain de Nice en milieu géographique contrasté : synthèse par approche inductive, climatologie, 10, 9-34.

Charfi S., 2012. Le comportement spatio-temporel de la 
température dans l'agglomération de Tunis. Thèse, Université de Tunis et Université de Nice (en cotutelle), 308 p.

Charfi S. et Dahech S., 2018. Cartographie des températures à Tunis par modélisation statistique et télédétection, Mappemonde, 123, $15 \mathrm{p}$.

Cantat O., 2004. L'îlot de chaleur urbain parisien selon les 'types de temps'. Norois, 191, 75-102.

Dahech S. \& Charfi S., 2017. Aspects topoclimatiques de Bizerte (NE de la Tunisie) : îlot de chaleur et brises thermiques. Les Impromptus, 138-158.

Dahech S. \& Ghribi M., 2017. Réchauffement climatique en ville et ses répercussions énergétiques. Méditerranée, 128, 29-38.

Dahech S., 2007. Le vent à Sfax (Tunisie) - Impact sur le climat et la pollution atmosphérique. Thèse doctorat, Université Paris VII, 351 p.

Dahech S., 2014. Impact de la brise de mer sur le confort thermique au Maghreb oriental durant la saison chaude. Cybergeo, European Journal of Geography Environnement, Nature, Paysage, $30 \mathrm{p}$.

Djen C. S., 1992. The urban climate of Shanghai. Atmospheric Environment, 26, 9-15.

Epstein Y. et S. Moran D., 2006. Thermal comfort and the heat stress indices. Industrial Health, 44, 388-398.

El Melki T., 2010. Brises du littoral et variation des concentrations polluées dans le grand Tunis : cas des banlieues de la Manouba et d'El Ghazela. 23 $3^{\text {ieme }}$ Colloque de l'Association Internationale de Climatologie, Rennes, 203208.

Ghribi M., 2012. Variation Spatio-temporelle des températures de surface en Tunisie. Mémoire de fin d'études, Université de Sfax, 58 p.

Hendel M. et al., 2017. Behavioral adaptation to heat-related health risks in cities. Energy and Buildings, 152, 823-829.

Hiemstra J. A., Saaroni H. \& Amorim J. H., 2017. The Urban Heat Island: Thermal Comfort and the Role of Urban Greening. The Urban Forest, 7-19.

INRS, 2004. Ambiances thermiques, travail en période de fortes chaleurs. Document pour le médecin de travail, 92, 5168.

Leconte F., 2014. Caractérisation des îlots de chaleur urbain par zonage climatique et mesures mobiles : Cas de Nancy. Thèse en climatologie, Université de Lorraine, 274 p.

Mathew A. et al., 2018. Analyzing the diurnal variations of land surface temperatures for surface urban heat island studies: Is time of observation of remote sensing data important? Sustainable Cities and Society, 40, 194-213.
Météo France, 2011. Conseils sur l'installation de capteurs météorologiques chez des passionnés. Note technique rédigée par Gaëtan Leches, 10 p.

Nassrallah W., 2019. Hydroclimatologie des zones urbaines dans les villes de Kairouan et de Sidi Bouzid : Approche intégrée du risque inondation. Thèse Université de Tunis et Université de Montpellier, 309 p.

Oke, T. R., 1988. Conception des rues et climat de la couche de canopée urbaine. Énergie et bâtiments, 11 (1-3), 103-113.

Qiuet G. et al., 2017. Experimental studies on the effects of green space and evapotranspiration on urban heat island in a subtropical megacity in China. Habitat International, 68, 3042.

Rothfusz L., 1990. The heat index equation. National Weather Service Technical Attachment (SR 90-23). 2 p.

Serrano S. M. V, Prats J. M. C., Sánchez M., 2015. Topography and vegetation cover influence on urban heat island of Zaragoza (Spain). 5th international conference on urban climate, $5 \mathrm{p}$.

Steadman R. G., 1979. The Assessment of Sultriness, Part II: Effects of Wind, Extra Radiation and Barometric Pressure on Apparent Temperature. Journal of Applied Meteorology, 18, 874-885.

Steadman R. G., 1984. A universal scale of apparent temperature. Journal of Climate and Applied Meteorology, 23 (12), 1674-1687.

Thom E. C., 1959. The discomfort index. Weather wise, 12, 57-60.

Wonga N., Jusuf S. K. et Tan C. L., 2011. Integrated urban microclimate assessment method as a sustainable urban development and urban design tool. Journal Landscape and Urban Planning, 100, 386-389.

Yang L., Qian F., Song D.-X. et Zheng K.-J., 2016. Research on Urban Heat-Island Effect. Procedia Engineering, 169, 1118.

Monteiro A., Carvalho V., Velho S. et Sousa C., 2013. The accuracy of the heat index to explain the excess of mortality and morbidity during heat waves - a case study in a Mediterranean climate. Bulletin of Geography, Socioeconomic Series, 20, Torun: Nicolaus Copernicus University Press, 71-84. DOI: 10.2478/bog-2013-0012.

Zoumakis M., Papadakis N., Benos A., Zoumakis N., Efstathiou G., Staliopoulou M., Tsoubaris P., Kassomenos P., Vosniakos F., Nikolaou K., 2012. Mortality and bioclimatic discomfort in the municipality of Thessaloniki, Greece. Protection and restoration of the environment, XI Conference, 1771-1784. 